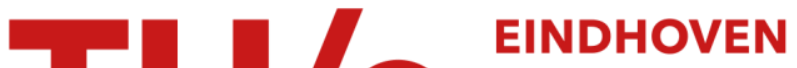

\section{Dynamics of prolate spheroidal elastic particles in confined shear flow}

Citation for published version (APA):

Villone, M. M., D'Avino, G., Hulsen, M. A., \& Maffettone, P. L. (2015). Dynamics of prolate spheroidal elastic particles in confined shear flow. Physical Review E - Statistical, Nonlinear, and Soft Matter Physics, 92(6), [062303]. https://doi.org/10.1103/PhysRevE.92.062303

DOI:

10.1103/PhysRevE.92.062303

Document status and date:

Published: 04/12/2015

\section{Document Version:}

Accepted manuscript including changes made at the peer-review stage

\section{Please check the document version of this publication:}

- A submitted manuscript is the version of the article upon submission and before peer-review. There can be important differences between the submitted version and the official published version of record. People interested in the research are advised to contact the author for the final version of the publication, or visit the $\mathrm{DOI}$ to the publisher's website.

- The final author version and the galley proof are versions of the publication after peer review.

- The final published version features the final layout of the paper including the volume, issue and page numbers.

Link to publication

\section{General rights}

Copyright and moral rights for the publications made accessible in the public portal are retained by the authors and/or other copyright owners and it is a condition of accessing publications that users recognise and abide by the legal requirements associated with these rights.

- Users may download and print one copy of any publication from the public portal for the purpose of private study or research.

- You may not further distribute the material or use it for any profit-making activity or commercial gain

- You may freely distribute the URL identifying the publication in the public portal.

If the publication is distributed under the terms of Article 25fa of the Dutch Copyright Act, indicated by the "Taverne" license above, please follow below link for the End User Agreement:

www.tue.nl/taverne

Take down policy

If you believe that this document breaches copyright please contact us at:

openaccess@tue.nl

providing details and we will investigate your claim. 


\title{
Dynamics of prolate spheroidal elastic particles in confined shear flow
}

\author{
M.M. Villone* \\ Center for Advanced Biomaterials for Health Care@CRIB, \\ Istituto Italiano di Tecnologia, Largo Barsanti e Matteucci 53, 80125 Napoli, Italy \\ G. D'Avino \\ Dipartimento di Ingegneria Chimica, dei Materiali e della Produzione Industriale, \\ Universitá di Napoli Federico II, P.le Tecchio 80, 80125 Napoli, Italy \\ M.A. Hulsen \\ Department of Mechanical Engineering, Eindhoven University of Technology, Eindhoven 5600 MB, The Netherlands
}

P.L. Maffettone

Dipartimento di Ingegneria Chimica, dei Materiali e della Produzione Industriale, Universitá di Napoli Federico II, P. le Tecchio 80, 80125 Napoli, Italy

\begin{abstract}
We investigate through numerical simulations the dynamics of a neo-Hookean elastic prolate spheroid suspended in a Newtonian fluid under shear flow. Both initial orientations of the particle within and outside the shear plane and both unbounded and confined flow geometries are considered. In unbounded flow, when the particle starts on the shear plane, two stable regimes of motion are found, i.e., trembling (TR), where the particle shape periodically elongates and compresses in the shear plane and the angle between its major semiaxis and the flow direction oscillates around a positive mean value, and tumbling (TU), where the particle shape periodically changes and its major axis performs complete revolutions around the vorticity axis. When the particle is initially oriented out of the shear plane, more complex dynamics arise. Geometric confinement of the particle between the moving walls also influences its deformation and regime of motion. In addition, when the particle is initially located in an asymmetric position with respect to the moving walls, particle lateral migration is detected. The effects on the particle dynamics of the geometric and physical parameters that rule the system are investigated.
\end{abstract}

PACS numbers: 47.57.E-, 83.50.-v, 47.11.Fg

\section{INTRODUCTION}

Soft particles, e.g., liquid droplets, elastic particles, biological cells, are often found in nature and technological applications. In particular, objects such as microgels and swollen starch granules can be regarded as elastic particles. In the last decades, the behavior of elastic particles has been investigated in the literature, aiming at determining their mechanical behavior in different circumstances.

When suspended in a flowing liquid, these systems deform under the action of the hydrodynamic forces exerted by the suspending medium. Since the early work by Roscoe [1], it is known that initially spherical elastic particles suspended in a Newtonian fluid subjected to shear flow attain a steady-state deformed shape with a fixed orientation with respect to the flow direction, the quantitative values of deformation and orientation being function of the flow conditions and the constitutive properties of the particle and the fluid. Gao and $\mathbf{H u}$ [2] performed a 2D perturbative analysis from which they derived a relationship between the steady elliptical deformation of an elastic particle and

* massimilianomaria.villone@unina.it the flow parameters, and validated such result by means of 2D finite element method simulations. Sugiyama et al. [3], instead, addressed the same topic in $2 \mathrm{D}$ through the finite difference method. Afterwards, Gao et al. [4] studied in 3D the behavior of an initially spherical elastic particle suspended in a Newtonian fluid under shear flow through a non-perturbative method, as developed in $[5,6]$, validating and extending Roscoe's results. In our previous works $[7,8]$, we studied through 3D finite element method numerical simulations the behavior of an initially spherical elastic particle suspended in Newtonian and viscoelastic fluids under unbounded and confined shear flow, validating the results in [1] and [4], and investigating the effects of matrix elasticity and geometrical confinement on the dynamics of the particle, in terms of deformation and lateral migration.

Non-spherical particles suspended in liquids are of great relevance in industrial applications and biological systems because of the great variety of dynamics arising from the non-triviality of their shape. A certain quantity of papers exist on the orientation dynamics of rigid ellipsoidal particles in Newtonian and non-Newtonian fluids under shear flow. Concerning Newtonian suspending media, Jeffery [9] extended Einstein's predictions for spheres to ellipsoidal particles under the same assumptions, finding that the forces acting on the surface of a rigid ellipsoid 
can be decomposed in two torques, one leading to a rotation around the vorticity axis and one making the particle spin around the flow direction. Thus, in a Newtonian fluid under unconfined shear flow, rigid ellipsoids indefinitely follow an orbit completely determined by the initial orientation. Several experimental papers confirmed such predictions [10-13]. For what matters non-spherical deformable particles, several studies exist on the behavior of spheroidal fluid vesicles and capsules suspended in Newtonian fluids under shear flow (for vesicles, see, for example, [14] and the references therein, whereas, for capsules, see [15] and the references therein). Depending on the flow parameters, spheroidal vesicles and capsules can undergo two different regimes of motion, namely, trembling (TR), where the particle periodically elongates and compresses and the angle between its major semiaxis and the flow direction oscillates around a mean positive value, and tumbling (TU), where the particle shape periodically changes and its major axis performs complete revolutions around the vorticity axis. In their recent paper, Gao et al. [16] have dealt with the behavior of a prolate spheroidal elastic particle in a Newtonian fluid under unbounded shear flow through the same method used in [4]. The authors have detected that, when the particle is initially placed with its major axis within the shear plane, such axis always lies on the shear plane, and the particle undergoes similar dynamics to the ones experienced by vesicles and capsules, i.e., TR or TU, depending on the flow parameters.

In this paper, the behavior of an elastic prolate spheroid suspended in unbounded and confined shear flow of a Newtonian liquid is studied by means of 3D arbitrary Lagrangian Eulerian finite element method numerical simulations. Both initial positions of the particle with its major axis within and outside the shear plane are investigated, yielding complicated deformation and orientation dynamics. Moreover, for the confined flow case, also asymmetric initial positions of the particle with respect to the moving walls are considered, yielding particle lateral migration. The effects of the geometric and physical parameters of the system on the deformation, the orientation, and the migration of the soft particle are investigated.

The paper is organized as follows: in Section II, the problem is presented; some hints on the numerical technique are given in Section III; in Section IV, the results are illustrated; finally, in Section V, some conclusions are drawn.

\section{PROBLEM OUTLINE}

The system under investigation is sketched in Fig. 1a: an elastic prolate spheroid is suspended in a Newtonian liquid under shear flow between two parallel plates. The center of the flow cell is coincident with the center of a
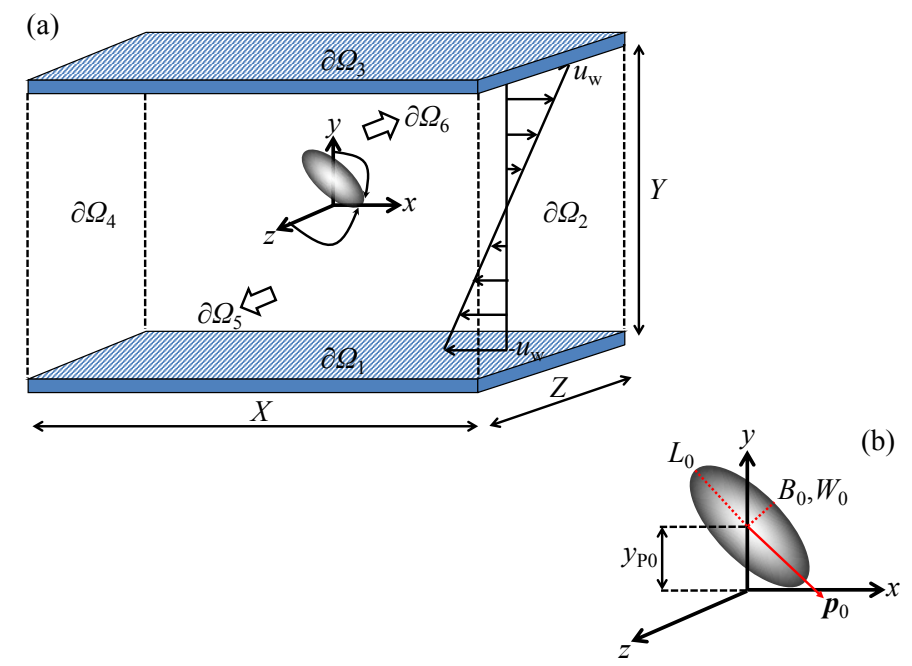

FIG. 1. a) Schematic drawing of an elastic prolate spheroid suspended in a Newtonian liquid under shear flow. b) Zoom on the spheroid, highlighting its initial geometry, position, and orientation.

Cartesian reference frame with its $x$-axis along the flow direction, the $y$-axis along the velocity gradient direction, and the $z$-axis along the vorticity direction. The center of volume of the particle has initial position [0, $\left.y_{\mathbf{P 0}}, \mathbf{0}\right]$, and the major axis of the spheroid, in general, lies out of the shear plane, i.e., the $x y$-plane in Fig. 1a. In Fig. 1b, a zoom of the spheroid is reported, aimed at highlighting its geometrical features: we denote with $L_{0}$ and $B_{0}\left(=W_{0}\right)$ the major and minor semiaxes of the undeformed spheroid, respectively, and we call the ratio between the two the 'aspect ratio' $A R=L_{0} / B_{0}$, which, then, measures the 'prolateness' of the spheroid and, in principle, can range between 1 (a sphere) and $\infty$ (a rod). The particle initial orientation is described by the orientation unit vector $\boldsymbol{p}_{0}=\left[p_{x 0}, p_{y 0}, p_{z 0}\right]$, that identifies the direction of the spheroid major semiaxis. In the general case, the particle is confined in the velocity gradient direction, which means that the blockage ratio $\beta=2 L_{0} / Y$ assumes a non-zero value; on the contrary, no confinement exists in the other directions.

For both the soft particle and the suspending phase, it is assumed that the inertia is negligible and that the material is incompressible, i.e., the volume is constant. Therefore, the mass and momentum balance for both phases read

$$
\nabla \cdot u=0
$$

and

$$
\nabla \cdot \sigma=0
$$

where $\boldsymbol{u}$ and $\boldsymbol{\sigma}$ are the velocity vector and the stress tensor, respectively. For the Newtonian liquid, $\boldsymbol{\sigma}$ can be, in turn, expressed as

$$
\boldsymbol{\sigma}=-p \boldsymbol{I}+2 \eta_{\mathrm{m}} \boldsymbol{D}
$$


where $p$ is the pressure, $\boldsymbol{I}$ is the identity tensor, $\eta_{\mathrm{m}}$ is the viscosity, and $\boldsymbol{D}$ is the symmetric part of the velocity gradient tensor $\left(\boldsymbol{D}=\frac{1}{2}\left(\nabla \boldsymbol{u}+\nabla \boldsymbol{u}^{\mathrm{T}}\right)\right)$. For the particle, $\boldsymbol{\sigma}$ can be written as

$$
\boldsymbol{\sigma}=-p \boldsymbol{I}+\boldsymbol{\tau}
$$

For the extra-stress tensor $\boldsymbol{\tau}$, we write

$$
\stackrel{\nabla}{\boldsymbol{\tau}}=2 G_{\mathrm{p}} \boldsymbol{D}
$$

which is the neo-Hookean elastic model with a modulus $G_{\mathrm{p}}$. The upper-convected derivative is defined by

$$
\stackrel{\nabla}{\boldsymbol{\tau}}=\dot{\boldsymbol{\tau}}-(\nabla \boldsymbol{u})^{T} \cdot \boldsymbol{\tau}-\boldsymbol{\tau} \cdot \nabla \boldsymbol{u}
$$

The balance equations that describe the system shown in Fig. 1a are supplied with the following boundary conditions:

$$
\begin{gathered}
\boldsymbol{u}=\left(-u_{\mathrm{w}}, 0,0\right) \text { on } \partial \Omega_{1} \\
\boldsymbol{u}=\left(u_{\mathrm{w}}, 0,0\right) \text { on } \partial \Omega_{3} \\
\left.\boldsymbol{u}\right|_{\partial \Omega_{2}}=\left.\boldsymbol{u}\right|_{\partial \Omega_{4}} \\
\left.\boldsymbol{\sigma} \cdot \boldsymbol{m}\right|_{\partial \Omega_{2}}=-\left.\boldsymbol{\sigma} \cdot \boldsymbol{m}\right|_{\partial \Omega_{4}} \\
\left.\boldsymbol{u}\right|_{\partial \Omega_{5}}=\left.\boldsymbol{u}\right|_{\partial \Omega_{6}} \\
\left.\boldsymbol{\sigma} \cdot \boldsymbol{m}\right|_{\partial \Omega_{5}}=-\left.\boldsymbol{\sigma} \cdot \boldsymbol{m}\right|_{\partial \Omega_{6}}
\end{gathered}
$$

Equations (7) and (8) are the adherence conditions on the matrix velocity on the lower and the upper walls of the flow cell, respectively; Equations (9) and (10) express the periodicity of velocity and stress in the matrix along the flow direction, with $\boldsymbol{m}$ the outwardly directed unit vector normal to the boundary; finally, Equations (11) and (12) are the periodical conditions on velocity and stress in the matrix along the vorticity direction.

The boundary conditions on the particle - matrix interface are

$$
\left.\boldsymbol{u}\right|_{\mathrm{m}}=\left.\boldsymbol{u}\right|_{\mathrm{p}}
$$

and

$$
\left(\left.\boldsymbol{\sigma}\right|_{\mathrm{m}}-\left.\boldsymbol{\sigma}\right|_{\mathrm{p}}\right) \cdot \boldsymbol{n}=\mathbf{0}
$$

where $\boldsymbol{n}$ is the outwardly directed unit vector normal to the interface. As the suspended particle is made of an elastic solid, no interfacial tension exists between it and the suspending fluid.

Since both the particle and the suspending medium are inertialess, no initial conditions on the velocities are required, whereas an initial condition is needed on the extra-stress tensor. We assume that the particle is initially stress-free, which means

$$
\left.\boldsymbol{\tau}\right|_{t=0}=\mathbf{0}
$$

The equations presented so far are made dimensionless by using the spheroid major semiaxis $L_{0}$ as the characteristic length, the inverse of the imposed shear rate $1 / \dot{\gamma}=Y / 2 u_{\mathrm{w}}$ as the characteristic time, $Y \dot{\gamma}$ as the characteristic velocity, $\eta_{\mathrm{m}} \dot{\gamma}$ as the characteristic stress in the matrix and the shear modulus of the elastic material $G_{\mathrm{p}}$ as the characteristic stress in the particle. The elastic capillary number, defined as $C a_{\mathrm{e}}=\eta_{\mathrm{m}} \dot{\gamma} / G_{\mathrm{p}}$, which is the ratio between the viscous forces and the elastic forces to which the particle is subjected, arises, then, from Equation (14). All the quantities that appear in the following Sections are made dimensionless through these characteristic quantities.

\section{NUMERICAL TECHNIQUE}

The equations presented in the previous Section are solved through the finite element method with an Arbitrary Lagrangian Eulerian (ALE) formulation. The numerical code makes use of stabilization techniques widely described in the literature, such as SUPG, DEVSS, logconformation [17-19].

Both the suspended particle and the suspending fluid domains are discretized by means of a mesh made of quadratic tetrahedra. On the particle-matrix interface, the mesh aligns with element faces (quadratic triangles), which are the same on the matrix and particle side (conforming geometry). The interface between the suspended particle and the suspending fluid needs to be tracked. A finite element method with second-order time discretization is defined on it: the normal velocity of the interface mesh equals the normal component of the fluid velocity, and the tangential velocity is such that the distribution of the elements on the interface is optimized. This approach lets the mesh get rid of the tank-treading motion of the particle, thus greatly reducing the distortion of the ALE volume mesh as compared to a Lagrangian description of the interface. In order to stabilize the interface, the SUPG method is used. A detailed description of the techniques, and, in particular, of the approach used on the interface, with several validation cases, is given in [7].

Due to the complex dynamics to which a spheroidal particle is subjected when suspended in shear flow, and to the presence of solid walls in the vicinity of the particle, during the simulations the elements of the volume mesh progressively warp; every time the mesh quality, in terms of the shape of the 'worst' element in the domain, goes below a fixed level, a remeshing is performed and the solution is projected from the old mesh to the new one.

Before running simulations, convergence tests have been performed in space and time, i.e., mesh resolution and time-step for the numerical solution of the equations 
proposed in Section II have been chosen that ensure invariance of the results upon further refinements. For the simulations presented in this paper, we have found that meshes with a number of tetrahedra in the order of $2-4 \cdot 10^{4}$ and time-steps in the order of $1-2 \cdot 10^{-3} \dot{\gamma}$ are adequate. A detailed description of the procedures adopted to run convergence tests for a problem similar to the one of our interest here is given in [7]. Furthermore, as periodicities are imposed in the flow and the vorticity directions, the $x$ and $z$-dimensions of the domain are chosen such that the particle does not 'feel' its periodic images along these directions: in all the cases shown in the following, the channel is 10 times the particle major semiaxis.

\section{RESULTS}

A neo-Hookean elastic prolate spheroid is suspended in a Newtonian fluid subjected to shear flow, as shown in Fig. 1.

\section{A. Unbounded shear flow}

Let us first consider the case of an unbounded flow geometry, i.e., $Y \gg L_{0}$ (or, in other words, $\beta \rightarrow 0$ ).

Fig. 2 reports the dynamics of a particle with aspect ratio $A R=1.1$ suspended with different initial orientations in a Newtonian fluid subjected to shear flow with elastic capillary number $C a_{\mathrm{e}}=0.2$. Due to the applied flow, the particle deforms, but, at variance to what happens to an initially spherical one [7], it does not reach a steady-state deformed shape and orientation.

When suspended with its major axis within the shear plane, as soon as the flow starts to act on the particle, the latter is no longer a spheroid, but it becomes a biaxial ellipsoid, i.e., an ellipsoid with all the three semiaxes of different lengths, namely, $L, B$, and $W$. (We recall that such lengths are made dimensionless through the initial length of the spheroid major semiaxis $L_{0}$.) The green curves in Fig. 2a display the temporal trends of $L$ (dashdot curve), $B$ (dashed curve), and $W$ (solid curve) for a spheroid initially oriented along the flow direction, i.e., with $\boldsymbol{p}_{0}=[1,0,0]$. It can be seen that, after an initial transient, the lengths of the three semiaxes oscillate with defined frequency and amplitude, which means that the particle periodically elongates and compresses under the action of the shear flow. In particular, it can be noticed that such frequency is about $1 / 2 \pi$.

In Fig. 3, the front $(x y)$ and top $(x z)$ views of the particle are displayed at $t \cong 3.6,5.2,6.8$, and 8.6. The first time-value $(t \cong 3.6)$ corresponds to an instant almost in the middle of the $L$-growth along a period (see the green curves in Fig. 2a); at $t \cong 5.2, L$ attains a maximum $(B$ and $W$ a minimum): in Fig. 3, it can be observed that this implies that the particle is maximally elongated in the shear plane; then, at $t \cong 6.8, L$ is almost in the

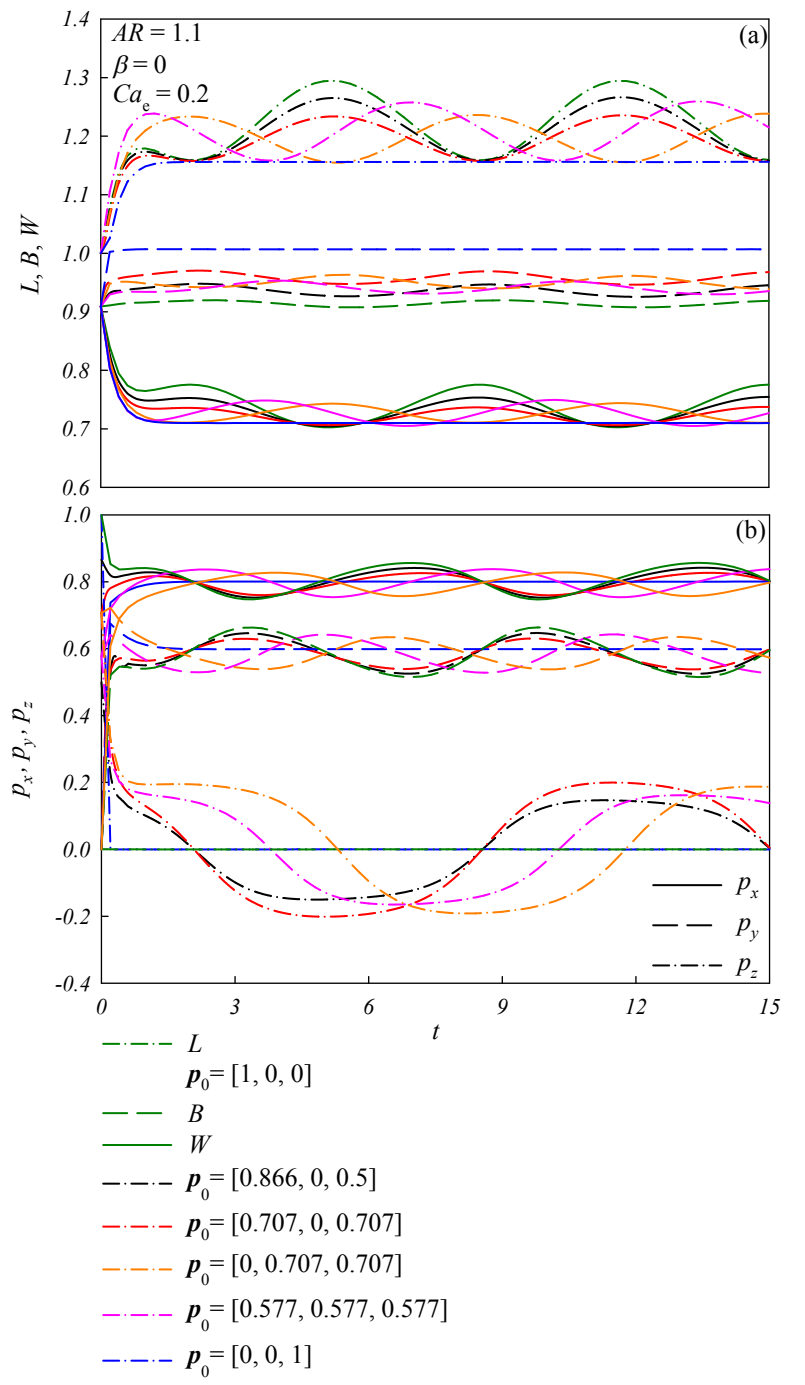

FIG. 2. (Color online) Dynamics of a neo-Hookean elastic prolate spheroid with $A R=1.1$ suspended with different initial orientations in a Newtonian fluid under unbounded shear flow with $C a_{\mathrm{e}}=0.2$. a) Time evolution of the ellipsoid dimensionless semiaxes $L, B, W$; b) Time evolution of the Cartesian components of the orientation unit vector $\boldsymbol{p}$.

middle of its decrease along a period, so the particle attains a very similar shape to the one attained at $t \cong 3.6$, though with a different orientation with respect to the flow; finally, at $t \cong 8.6, L$ attains a minimum ( $B$ and $W$ a maximum), so the particle is maximally 'compressed'. Such shape dynamics recurs periodically in time.

The green curves in Fig. 2b report the temporal trends of the Cartesian components $p_{x}, p_{y}$, and $p_{z}$ of the orientation vector of the spheroid initially placed along the flow direction. As $p_{z}$ does not move from 0 (see the green dash-dot line), the particle orientation vector always lies on the shear plane, with its $x$ - and $y$-components (represented by the green solid and dashed lines, respectively) 


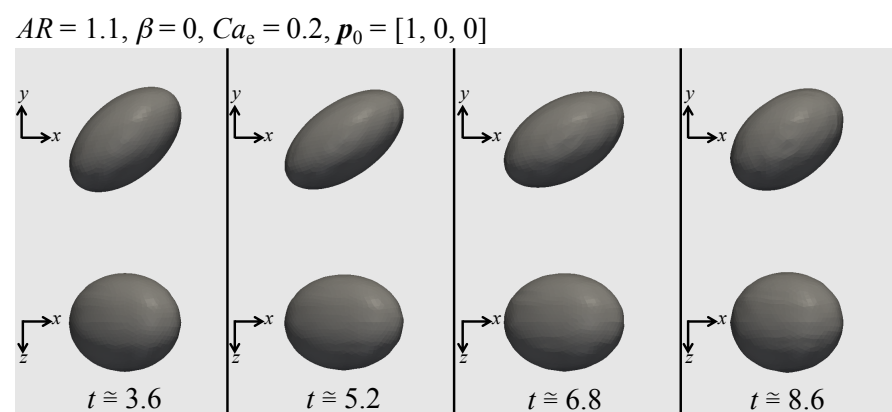

FIG. 3. Shape evolution of a neo-Hookean elastic prolate spheroid with $A R=1.1$ suspended with initial orientation $\boldsymbol{p}_{0}=[1,0,0]$ in a Newtonian fluid under unbounded shear flow with $C a_{\mathrm{e}}=0.2$. The shapes attained by the particle at four dimensionless times are displayed, i.e., $t \cong 3.6,5.2,6.8,8.6$. Top: front views; bottom: top views.

that, after a brief initial transient, oscillate with defined amplitude and frequency, always assuming positive values. In other words, the particle major semiaxis forms an angle with the flow direction that is always positive and oscillates around a mean value. When the particle is initially placed in the shear plane, the numerical value of the initial orientation has no effects on the 'quality' of the particle dynamics, but only introduces a time-shift. Such behavior, known as trembling (TR), has been also found by Gao et al. [16] for the same particle initial shape and the same flow conditions.

When the spheroid is suspended with its major axis out of the shear plane, during its dynamics, it is no longer a spheroid and, strictly speaking, it is neither an ellipsoid. However, since the departures from 'ellipsoidity' are not great, we still consider the semiaxes $L, B$, and $W$ of the ellipsoid that best fits the particle deformed shape. Analogously, we still measure the particle orientation through the unit vector $\boldsymbol{p}$. In Fig. 2, besides the case with $\boldsymbol{p}_{0}=[1,0,0]$ discussed above, the results for several initial orientations out of the shear plane are presented. The distribution of these orientations is such that the whole octant of the Cartesian space characterized by positive $x$-, $y$ - and $z$-values is investigated, the dynamics in the other octants being analogous due to symmetry reasons. By looking at the black $\left(\boldsymbol{p}_{0}=[0.866,0,0.5]\right)$, red $\left(\boldsymbol{p}_{0}=[0.707,0,0.707]\right)$, orange $\left(\boldsymbol{p}_{0}=[0,0.707,0.707]\right)$, and pink $\left(\boldsymbol{p}_{0}=[0.577,0.577,0.577]\right)$ curves in Figs. 2a, $\mathrm{b}$, and $\mathrm{c}$, an analogous qualitative behavior can be detected; indeed, for all these four starting positions, the particle dynamics is characterized by shape and orientation oscillations. It is worth noticing that the frequency of such oscillations is almost invariant with respect to the particle initial orientation and has a value of about $1 / 2 \pi$. For what matters orientation dynamics, from the $p_{x^{-}}$and $p_{y}$-trends, it is evident that the particle major semiaxis oscillates always staying above the flow-vorticity plane, whereas, from the $p_{z}$-trend, it can be seen that, during its oscillations, the particle continuously crosses the flowgradient plane. A 'special' case is the spheroid initially oriented along the vorticity, i.e., with $\boldsymbol{p}_{0}=[0,0,1]$, whose dynamics is represented by the blue curves in Fig. 2: such particle, whose cross-section in the flow-gradient plane is initially circular, 'squeezes' in the $z$-direction, whereas it elongates orthogonally to it, until it attains a steadystate deformed shape and orientation with respect to the flow. By looking at Fig. 2b, it can be seen that the $x$ and $y$-components of $\boldsymbol{p}$ start from 0 and reach a non-zero value, whereas the $z$-component starts from 1 and decays to zero: this means that the flow 'strength' is such that the squeezing in the $z$-direction makes the particle major semiaxis 'change' from being aligned with the z-axis to lying on the $x y$-plane.

In order to better visualize the orientation dynamics of the particle, in Fig. 4 the projections on the flowgradient (panel a) and flow-vorticity (panel b) planes of the trajectories traced by the unit vector $\boldsymbol{p}$ are shown for $A R=1.1, C a_{\mathrm{e}}=0.2$, and for four initial orientations already considered in Fig. 2, i.e., $\boldsymbol{p}_{0}=[0,0.707,0.707]$ (orange curves), [0.577, 0.577, 0.577] (pink curves), [0.707, $0,0.707]$ (red curves), and $[1,0,0]$ (green curves). In both panels, each initial orientation of the particle is symbolized by a circle of the same color of the trajectory, and an arrow identifies the direction of travel of the trajectory. The orientation dynamics of the particle initially placed within the shear plane $\left(\boldsymbol{p}_{0}=[1,0,0]\right)$ follows the aforementioned TR motion: by looking at the projection of the unit vector $\boldsymbol{p}$ on the $x y$-plane (panel a), it can be seen that, after an initial transient, $\boldsymbol{p}$ goes back-andforth along the same trajectory; similarly, by looking at panel b, it can be observed that the projection of $\boldsymbol{p}$ on the $x z$-plane goes back-and-forth on a portion of the $x$ axis. When initial orientations out of the shear plane are considered (orange, pink, and red curves), the following common dynamics happen: after an initial transient, the $x y$-projection of $\boldsymbol{p}$ 'draws' arcs of increasing amplitude, whereas its $x z$-projection oscillates symmetrically about the $x$-axis, roughly tracing the number ' 8 '. Such kind of behavior has been recently documented by Cordasco and Bagchi [20] for prolate spheroidal capsules in shear flow of a Newtonian fluid, and it is referred as 'kayaking'. As visible in Fig. 4b, the amplitude of the 'eyes' of the 8 -s progressively decreases with respect to the $z$-axis and increases with respect to the $x$-axis, thus attesting that, regardless $\boldsymbol{p}_{0}$, at long (in principle, infinite) times, the dynamics of a particle starting out of the shear plane will conform to the TR motion of a spheroid initially placed with its major axis on the shear plane, except the very special case with $\boldsymbol{p}_{0}=[0,0,1]$ (see the blue curves in Fig. 2 ). Therefore, for $A R=1.1$ and $C a_{\mathrm{e}}=0.2$, TR is the stable regime towards which the particle tends from every ini- 


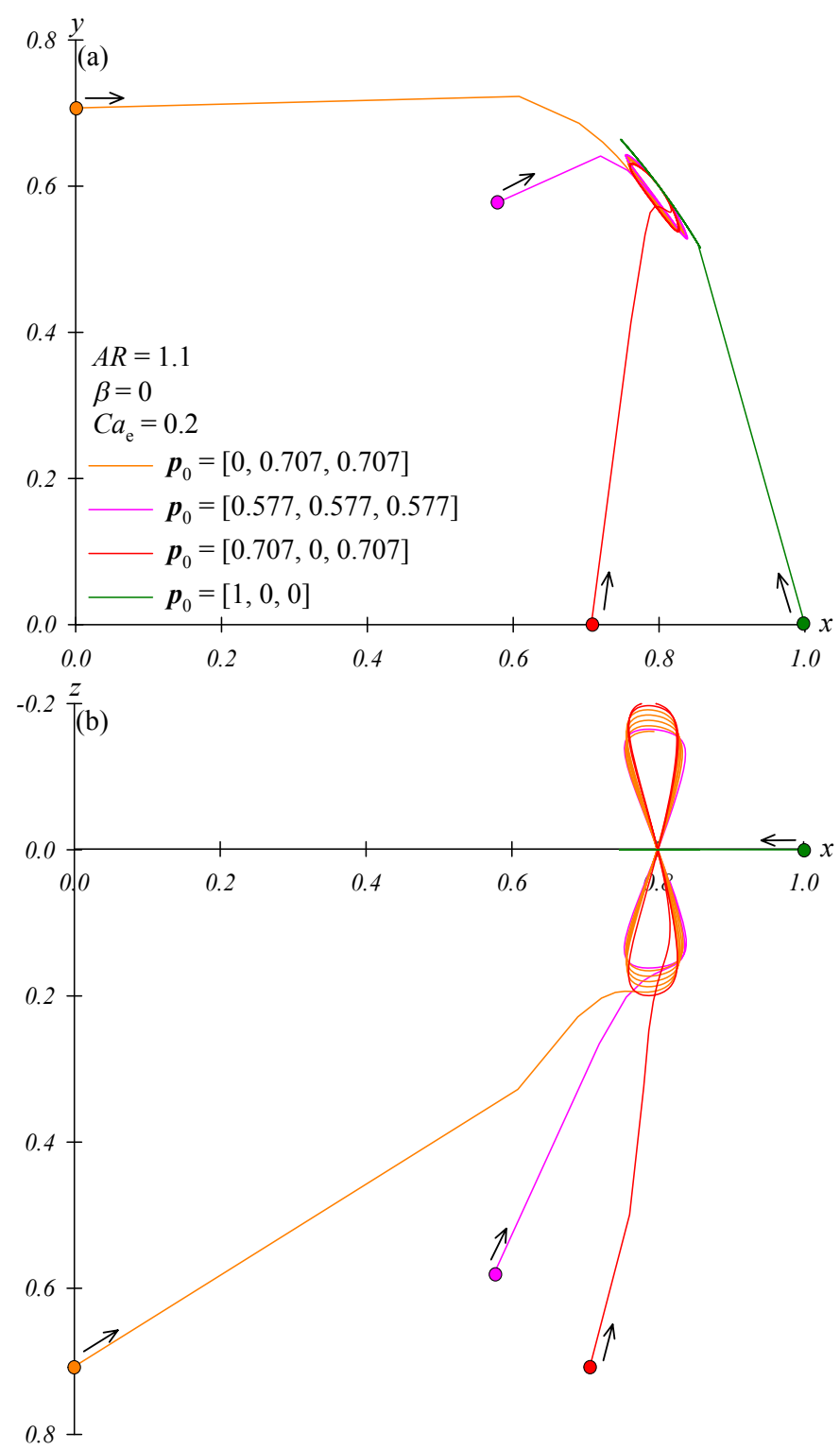

FIG. 4. (Color online) Dynamics of a neo-Hookean elastic prolate spheroid with $A R=1.1$ suspended with different initial orientations in a Newtonian fluid under unbounded shear flow with $C a_{\mathrm{e}}=0.2$. a) Projection on the $x y$-plane of the trajectories traced by the orientation unit vector $\boldsymbol{p}$; b) Projection on the $x z$-plane of the trajectories traced by the orientation unit vector $\boldsymbol{p}$. In both panels, the circles represent the particle initial orientations and the arrows indicate the direction of travel of the curves.

tial orientation, the steady-state solution for $\boldsymbol{p}_{0}=[0,0,1]$ being, actually, unstable, since even a little perturbation which makes the particle move from its steady-state position would make it go towards the periodic regime. It is worth remarking that the crossing of the shear-gradient plane performed by the particles starting outside such plane would be impossible for a rigid ellipsoid in a Newtonian fluid under shear flow [9]: its occurrence in the present case is likely due to the mechanism of torque dissipation inside a deformable solid, which makes the ellipsoid oscillate about the $x y$-plane before finally lying on it.
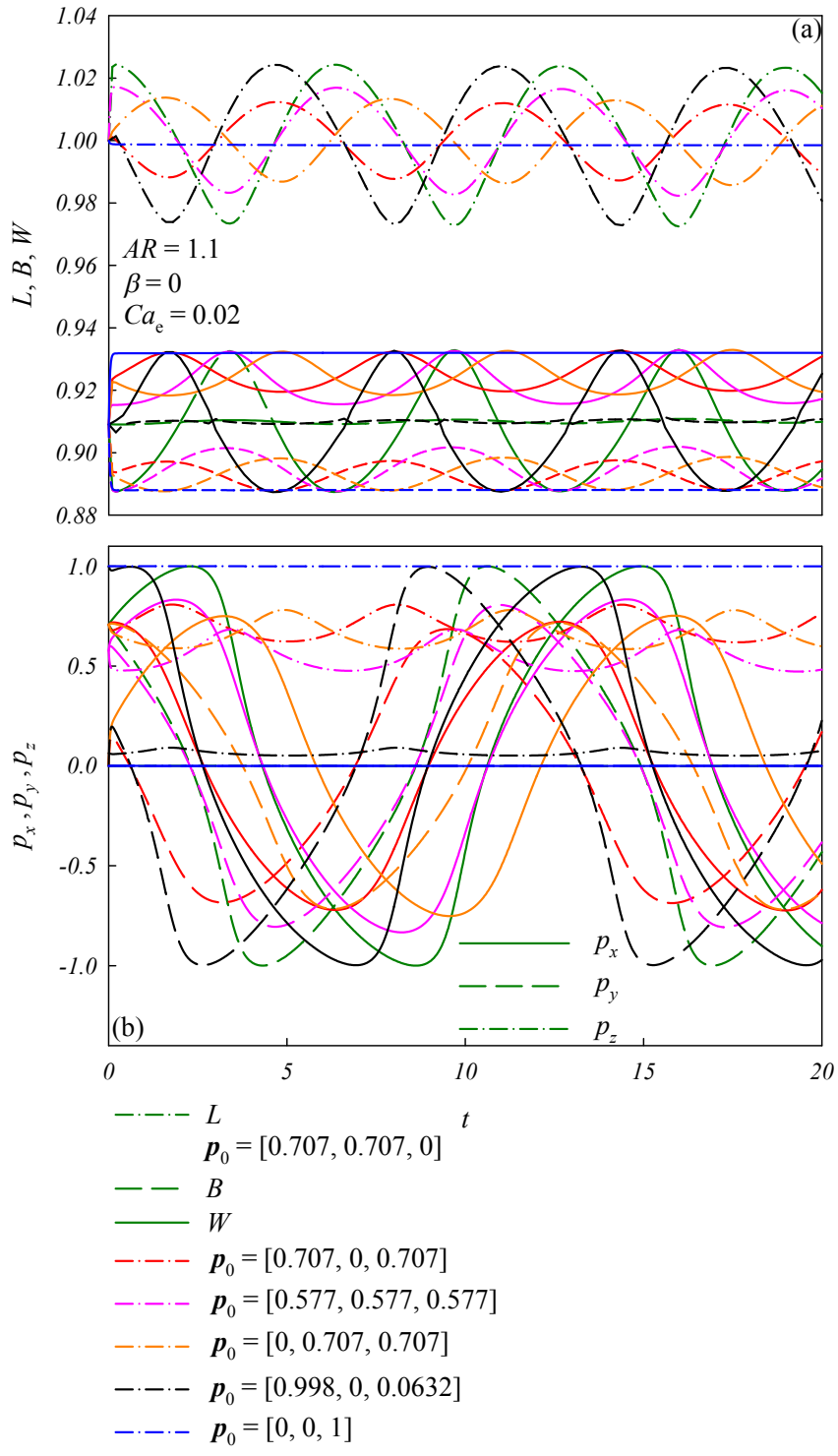

FIG. 5. (Color online) Dynamics of a neo-Hookean elastic prolate spheroid with $A R=1.1$ suspended with different initial orientations in a Newtonian fluid under unbounded shear flow with $C a_{\mathrm{e}}=0.02$. a) Time evolution of the ellipsoid dimensionless semiaxes $L, B, W$; b) Time evolution of the Cartesian components of the orientation unit vector $\boldsymbol{p}$.

Let us investigate the effect of the elastic capillary number $C a_{\mathrm{e}}$ on the behavior of a particle with aspect ratio $A R=1.1$ suspended with different initial orientations in a Newtonian fluid subjected to unbounded shear flow. In Fig. 5, the dynamics of such particle is reported for $C a_{\mathrm{e}}=0.02$. 
The green curves in Fig. 5a display the temporal trends of $L$ (dash-dot line), $B$ (dashed line), and $W$ (solid line) for a spheroid initially placed within the shear plane with $\boldsymbol{p}_{0}=[0.707,0.707,0]$. After an initial transient, the semiaxes lengths begin to oscillate with defined frequency (again, equal to about $1 / 2 \pi$ ) and amplitude. The green curves in Fig. 5b report the temporal trends of the Cartesian components $p_{x}, p_{y}$, and $p_{z}$ of the orientation vector of the spheroid. Like for $C a_{\mathrm{e}}=0.2$ (see Fig. 2b), $p_{z}$ (green dash-dot line) does not move from 0 , so the particle orientation vector alway lies on the shear plane. After a brief initial transient, its $x$ - and $y$-components (green solid and dashed lines, respectively) oscillate with defined amplitude and frequency between -1 and +1 ; this means that the particle major semiaxis performs periodical revolutions around the vorticity axis (i.e., the $z$-axis). Such behavior is known as tumbling (TU), and it has been also found by Gao et al. [16] for the same particle initial shape and flow conditions. In Fig. 5, the results for some initial orientations out of the shear plane are also shown. As for the case with $C a_{\mathrm{e}}=0.2$ discussed above, the distribution of the orientations is such that the whole octant of the Cartesian space characterized by positive $x-, y$ and $z$-values is investigated, the dynamics in the others being analogous due to symmetry. For all the starting positions out of the shear plane considered here, the particle dynamics is characterized by shape and orientation oscillations. In particular, the $x$ - and $y$-components of the orientation vector $\boldsymbol{p}$ oscillate with increasing peak heights, whereas its $z$-component oscillates with decreasing peak heights, always staying positive. As above, a 'special' case is represented by the spheroid with $\boldsymbol{p}_{0}=[0$, $0,1]$, that, after a transient, attains a steady-state deformed shape with respect to the flow, as shown by the blue lines representing $L, B$, and $W$ in Fig. 5a. At variance with the case with $C a_{\mathrm{e}}=0.2$, here the orientation of the particle does not change, as $p_{x}$ and $p_{y}$ do not move from 0 , and $p_{z}$ does not move from 1 , so the particle keeps its initial orientation along the vorticity.

In order to highlight the dynamics of the particle orientation, in Fig. 6 the projections on the flow-gradient (panel a) and flow-vorticity (panel b) planes of the trajectories traced by the unit vector $\boldsymbol{p}$ are shown for $A R=1.1$, $C a_{\mathrm{e}}=0.02$, and for $\boldsymbol{p}_{0}=[0.707,0.707,0]$ (green curves), [0.707, 0, 0.707] (red curves), [0.577, 0.577, 0.577] (pink curves), [0, 0.707, 0.707] (orange curves), and [0.998, 0, 0.0632] (black curves). Like in Fig. 4, each particle initial orientation is represented by a circle of the same color of the trajectory, and an arrow identifies the direction of travel of the trajectory. The particle initially lying on the shear plane $\left(\boldsymbol{p}_{0}=[0.707,0.707,0]\right)$ undergoes TU motion: by looking at panel a, it can be noticed that the projection of $\boldsymbol{p}$ moves clockwise along a circular trajectory; consequently, from panel b it appears that the projection of $\boldsymbol{p}$ on the shear-vorticity plane goes back-and-forth along the $x$-axis. When initial orientations out of the shear plane are considered (red, pink, orange, and black curves), the following qualitative dynamics happen: the
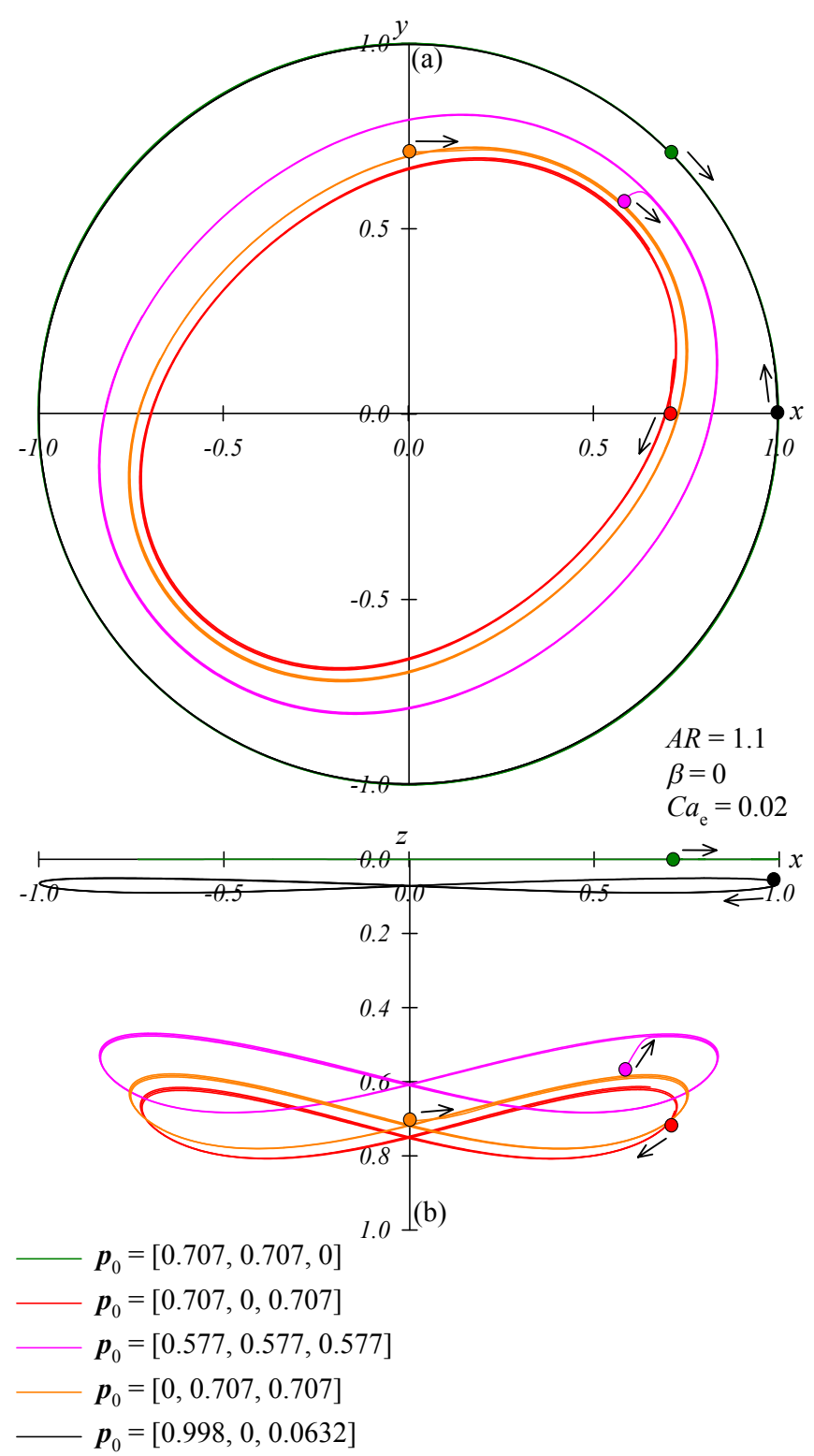

FIG. 6. (Color online) Dynamics of a neo-Hookean elastic prolate spheroid with $A R=1.1$ suspended with different initial orientations in a Newtonian fluid under unbounded shear flow with $C a_{\mathrm{e}}=0.02$. a) Projection on the $x y$-plane of the trajectories traced by the orientation unit vector $\boldsymbol{p}$; b) Projection on the $x z$-plane of the trajectories traced by the orientation unit vector $\boldsymbol{p}$. In both panels, the circles represent the particle initial orientations and the arrows indicate the direction of travel of the curves.

projection of $\boldsymbol{p}$ on the $x y$-plane has a spiral-like trajectory with increasing amplitude, whereas its $x z$-projection oscillates drawing 'horizontal 8-s' with increasing amplitude and decreasing $z$-component. Such behavior is somehow opposite to those documented in [20] for prolate spheroidal capsules in shear flow of a Newtonian fluid 
and in [21] for rigid prolate spheroids in shear flow of a viscoelastic fluid, where the particle has a spiraling motion tending to a vorticity-alignment. Here, on the contrary, at long times, the dynamics of a particle starting out of the shear plane will conform to TU regardless its $\boldsymbol{p}_{0}$-value (except the case with $\left.\boldsymbol{p}_{0}=[0,0,1]\right)$. Thus, for $A R=1.1$ and $C a_{\mathrm{e}}=0.02$, TU is the regime towards which the particle tends from every initial orientation, except the unstable steady-state solution for $\boldsymbol{p}_{0}=[0,0,1]$.

Hence, we are now in the position to say that, fixed the aspect ratio $A R$ of the prolate spheroid, 'low' values of the elastic capillary number $C a_{\mathrm{e}}$ promote $\mathrm{TU}$, whereas 'high' $C a_{\mathrm{e}}$-values promote TR. We have also investigated the effect of $A R$ on the dynamics of an elastic prolate spheroid suspended in a Newtonian fluid subjected to unbounded shear flow at fixed $C a_{\mathrm{e}}$. As we have detected the very same qualitative behaviors illustrated so far, both for particles initially oriented within and outside the shear plane, these are not shown. We just report that increasing $A R$ promotes $\mathrm{TU}$, whereas decreasing it promotes TR.

\section{B. Confined shear flow}

\section{Particle suspended symmetrically between the moving walls}

Let us, now, investigate the case where the spheroid is suspended with its center of volume in a symmetric position with respect to the two moving walls, i.e., with $y_{\mathbf{P 0}}=0$. In turn, the above mentioned walls are placed at a finite distance $Y$, which means that the blockage ratio $\beta$ assumes non-zero values. For the sake of simplicity, only results for a particle with its major axis initially lying on the shear plane are shown below, the dynamics that the particle can possibly undergo when starting with its major axis out of the shear plane being qualitatively similar to the ones shown in Sec. IV A for an unbounded flow geometry.

Fig. 7 reports the dynamics of a particle with $A R=1.25$ suspended with initial orientation $\boldsymbol{p}_{0}=[0.707,0.707,0]$ in a Newtonian fluid subjected to confined shear flow with elastic capillary number $C a_{\mathrm{e}}=0.06$ and for 5 different values of the blockage ratio, i.e., $\beta=0$ (unbounded flow), $0.25,0.50,0.66,0.75$. Like in unbounded flow, the particle deforms under the action of the sheared suspending fluid. During the particle dynamics, the orientation unit vector $\boldsymbol{p}$ always lies in the $x y$-plane due to symmetry reasons. Therefore, the particle orientation can be described by a scalar parameter, namely, the angle $\theta$ between the flow direction and the unit vector $\boldsymbol{p}$, to which we will refer as to the 'orientation angle'. Since during its dynamics the particle shape never departs from a (biaxial) ellipsoid, the semiaxes $L, B$, and $W$ are considered to evaluate the particle deformation in time. It can be seen in Fig. 7a that, after an initial transient, $L, B$, and $W$ undergo periodic oscillations for all the blockage ratios
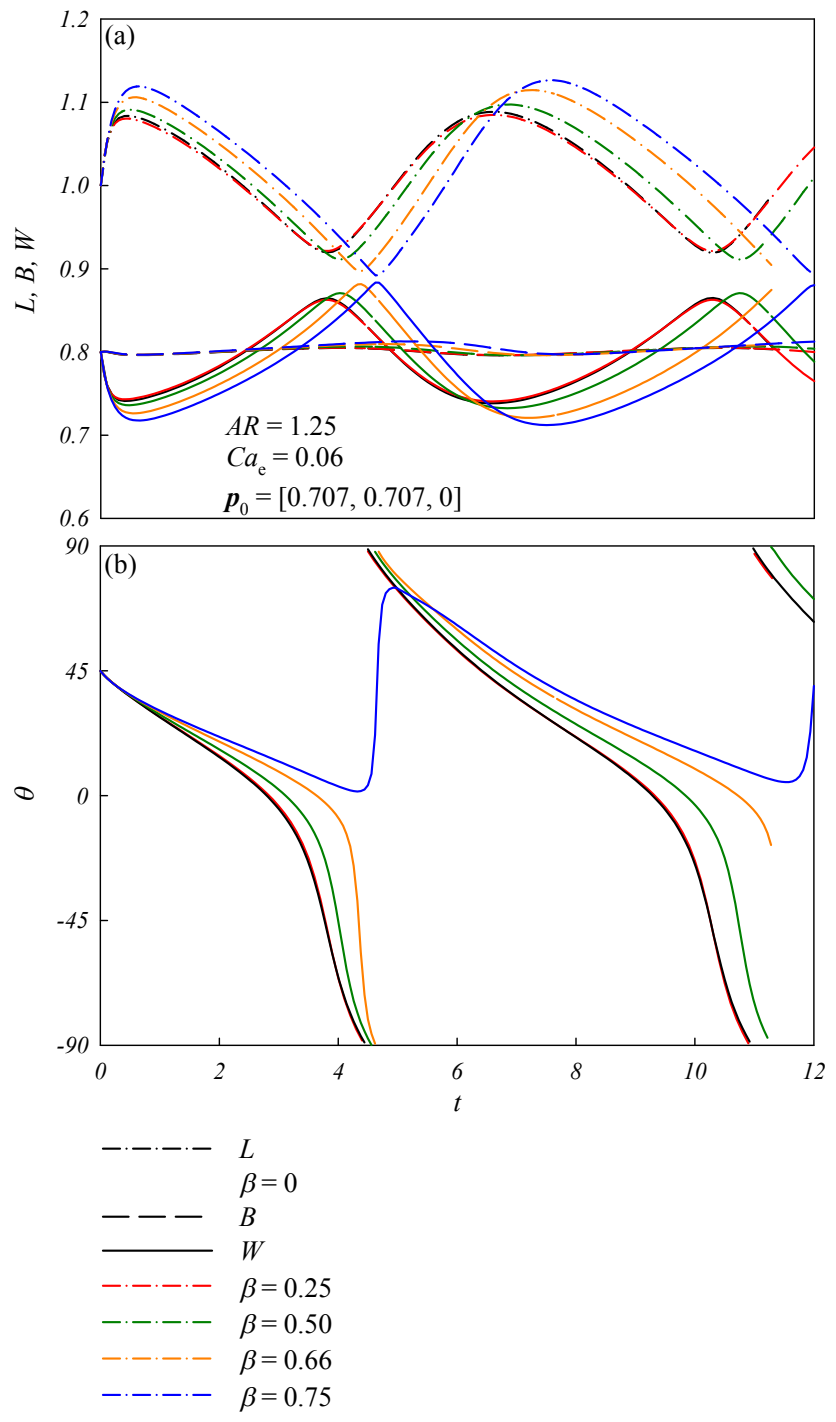

FIG. 7. (Color online) Dynamics of a neo-Hookean elastic prolate spheroid with $A R=1.25$ suspended in a Newtonian fluid under confined shear flow for $C a_{\mathrm{e}}=0.06$ and 5 different values of the blockage ratio $\beta$. In all cases, the particle initial orientation is $\boldsymbol{p}_{0}=[0.707,0.707,0]$. a) Time evolution of the ellipsoid dimensionless semiaxes $L, B, W$; b) Time evolution of the orientation angle $\theta$.

considered. For all the three semiaxes, the amplitude of these oscillations increases with $\beta$, the numerical values of their maxima increase with $\beta$, and the minima decrease with $\beta$; in other words, the more the particle is confined between the moving walls, the more it stretches and compresses under the action of the suspending liquid. Yet the most interesting effect of confinement on the particle behavior can be caught from Fig. 7b. At increasing $\beta$ from 0 to 0.66 , the ellipsoid major axis is found to perform complete revolutions around the vorticity axis, i.e., the particle undergoes $\mathrm{TU}$; then, by further increasing $\beta$, a 
transition in the regime of motion is detected: indeed, as clearly visible from Fig. $7 \mathrm{~b}$, for $\beta=0.75$, the orientation angle $\theta$ oscillates always staying positive, which means that the particle is subjected to TR.

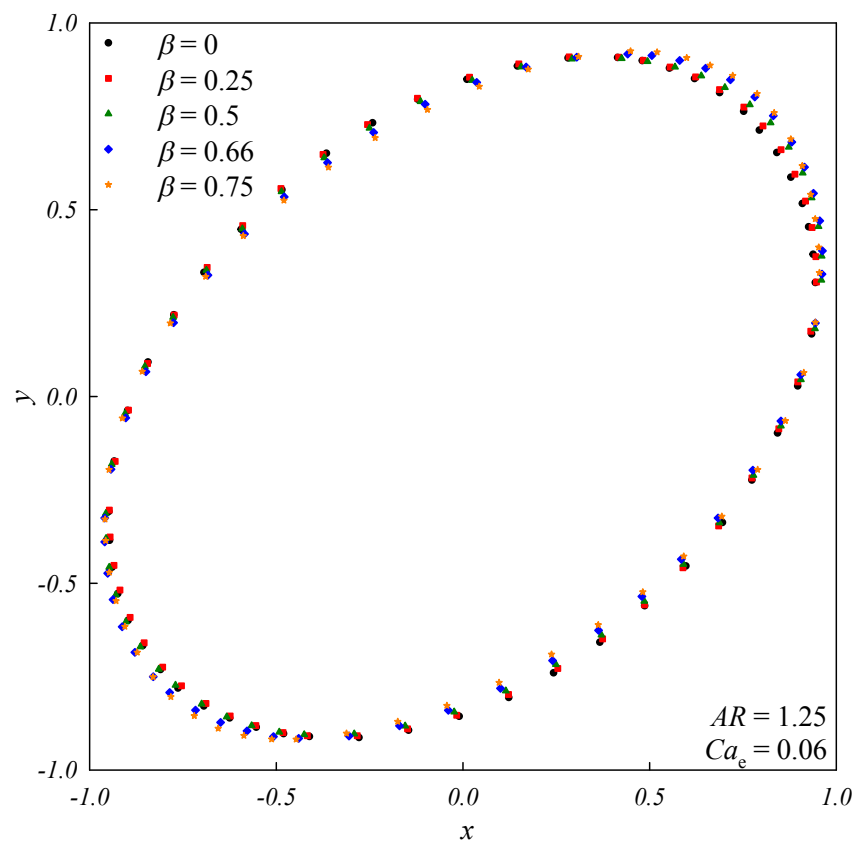

FIG. 8. (Color online) Projection on the $x y$-plane of the maximally elongated shape of a neo-Hookean elastic prolate spheroid with $A R=1.25$ suspended in a Newtonian fluid under confined shear flow for $C a_{\mathrm{e}}=0.06$ and 5 different values of the blockage ratio $\beta$.

In Fig. 8, the projection on the shear-gradient plane of the particle deformed shape in correspondence of the maximum $L$-value is reported for $\beta=0,0.25,0.50,0.66$, 0.75 . As mentioned above, by increasing the blockage ratio $\beta$, the particle maximum deformation increases. It is interesting to notice that, regardless the blockage ratio (and, consequently, the regime of motion), when the particle attains the highest deformation, it is always oriented in the same way with respect to the flow. In particular, for the case under investigation (i.e., for $A R=1.25$ and $\left.C a_{\mathrm{e}}=0.06\right)$, the orientation angle corresponding to the maximum elongation is equal to about $41^{\circ}$. This can be read quantitatively from Fig. 7 : for each $\beta$, if one puts in the maximum $L$ in panel a and, then, goes down to panel $\mathrm{b}$ until intersecting the $\theta$-curve at the same $\beta$-value, always the same value of the orientation angle can be read on the $y$-axis.

The effects of the interplay of the three parameters that rule the system, i.e., the aspect ratio $A R$, the elastic capillary number $C a_{\mathrm{e}}$, and the blockage ratio $\beta$, on the regime of motion of a prolate spheroid suspended with its major axis on the shear plane are depicted in the phase diagram displayed in Fig. 9. The black solid curve reports the non-perturbative predictions from [16]

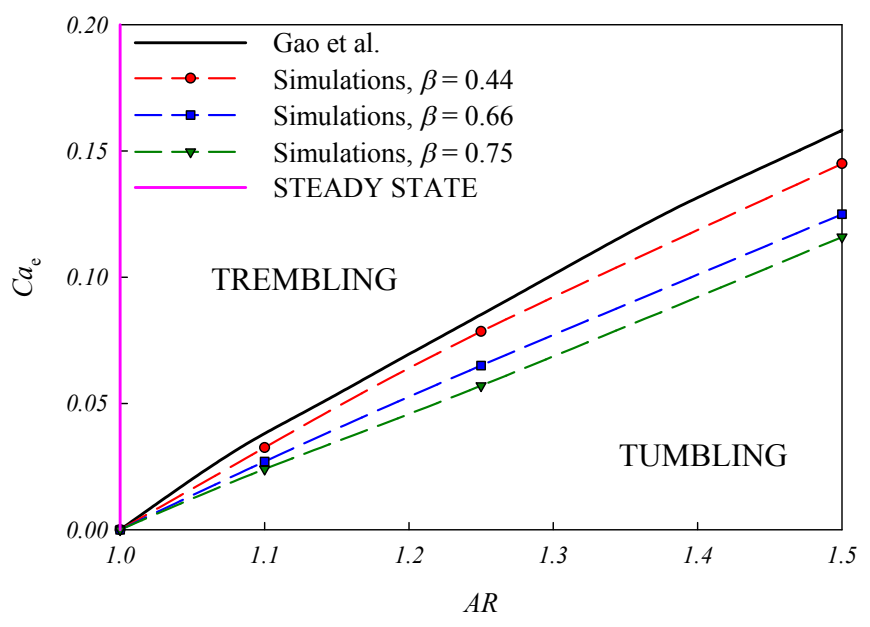

FIG. 9. (Color online) Phase diagram for a neo-Hookean elastic prolate spheroid suspended in a Newtonian fluid under shear flow. The black solid curve shows theoretical predictions from [16], the dashed lines and symbols represent our numerical simulation results.

for an unbounded flow geometry (with which our numerical data quantitatively agree), whereas the series of red, blue and green symbols connected through dashed lines of the same color report our numerical simulation results at varying $\beta$ (red corresponds to $\beta=0.44$, blue to $\beta=0.66$, and green to $\beta=0.75$ ). For each of the considered blockage ratios, every $A R-C a_{\mathrm{e}}$ couple below the dashed curve identifies a TU condition; on the contrary, every $A R-C a_{\mathrm{e}}$ couple above such curve identifies a TR condition. Fixed $\beta$ and $A R$, increasing $C a_{\mathrm{e}}$ is found to promote TR; fixed $\beta$ and $C a_{\mathrm{e}}$, increasing $A R$ pushes the system towards TU; finally, fixed $A R$ and $C a_{\mathrm{e}}$, more confined geometries (in other words, increasing $\beta$ ) promote TR. A very special case is represented by spheroids with $A R=1$, that are, actually, spheres: when suspended in shear flow, initially spherical elastic particles deform until they attain a steady-state deformation and orientation with respect to the flow (in this regard, see [8]). In the phase diagram shown in Fig. 9, the locus of such 'special' solutions coincides with the $y$ axis, as highlighted by the pink curve. As soon as the particle undeformed shape departs from a sphere, no steady-state solutions are found to exist, as also reported in $[16]$.

\section{Particle suspended asymmetrically between the moving walls}

Finally, we address the case of a spheroid with its center of volume initially placed in an asymmetric position with respect to the two moving walls, i.e., with $y_{\mathbf{P 0}} \neq 0$. As in section IV B 1, the walls are at a finite distance $Y$, thus the blockage 
ratio $\beta$ attains non-zero values.

Due to the asymmetry of the spheroid initial position with respect to the walls of the flow cell, a new phenomenon arises, namely, the migration of the particle orthogonally to the flow direction along the $y$-direction. In what follows, when we refer to the 'vertical position' of the particle $y_{\mathbf{P}}$, we will mean the $y$-coordinate of its center of volume; similarly, when we mention the 'migration velocity' of the particle, we will mean the $y$ component of the translational velocity computed in its center of volume.

In Fig. 10a, the trajectories of the particle are displayed for an aspect ratio $A R=1.25$, two values of the blockage ratio $(\beta=0.25,0.5)$, two values of the elastic capillary number $\left(C a_{\mathbf{e}}=\right.$ $0.06,0.2)$, two initial orientations of the spheroid, one in the shear plane and one outside it $\left(p_{0}=\right.$ $[0.707,0.707,0],[0.577,0.577,0.577])$, and several initial vertical positions $y_{\mathrm{P} O}$ in the upper half of the channel, the dynamics in the lower half being analogous (mirrored) due to symmetry. In order to report results at different blockage ratios on the same graph, the value of the particle vertical position $y_{\mathrm{P}}$ is normalized by dividing it by the value of the maximum vertical position attainable by the particle center of volume $Y / 2-L_{0}$. By looking at Fig. 10a, it can be seen that, whatever the values of the parameters, the particle migrates towards the center plane of the channel, that is the $x z$-plane at $y=0$. More in detail, the slopes of the curves displayed in the above mentioned figure show that both increasing $\beta$ and $C a_{\mathbf{e}}$ promotes a faster lateral migration; on the other hand, fixed $\beta$ and $C a_{\mathbf{e}}$, a different initial orientation does not induce appreciable differences on the trajectories (see the pink-orange, black-red, and green-blue couples of curves). In Fig. 10b, we plot the particle migration velocity $v_{\mathrm{P}} v s$. its normalized vertical position in the upper semichannel $y_{\mathbf{P}} /\left(Y / 2-L_{0}\right)$ for the same parameters considered in Fig. 10a. For every set of parameters, the migration velocity trends show that, as the particle travels towards the channel center plane, its migration velocity decreases (in magnitude) through damping oscillations, which can be linked to particle shape and orientation oscillations (see Fig. 12). Fixed a set of $\beta-, C a_{\mathbf{e}^{-}}$, and $p_{0}$-values, it can be seen that the curves corresponding to different particle initial positions all arrange along a 'master' trend, even if different starting positions can induce a phase shift in the damping oscillations, due to the presence of an initial velocity-transient. We remark that the migration velocity steep descents appearing in Fig. 10b correspond, for each particle starting position, to a time span in which the particle has an abrupt deformation with respect to the initial
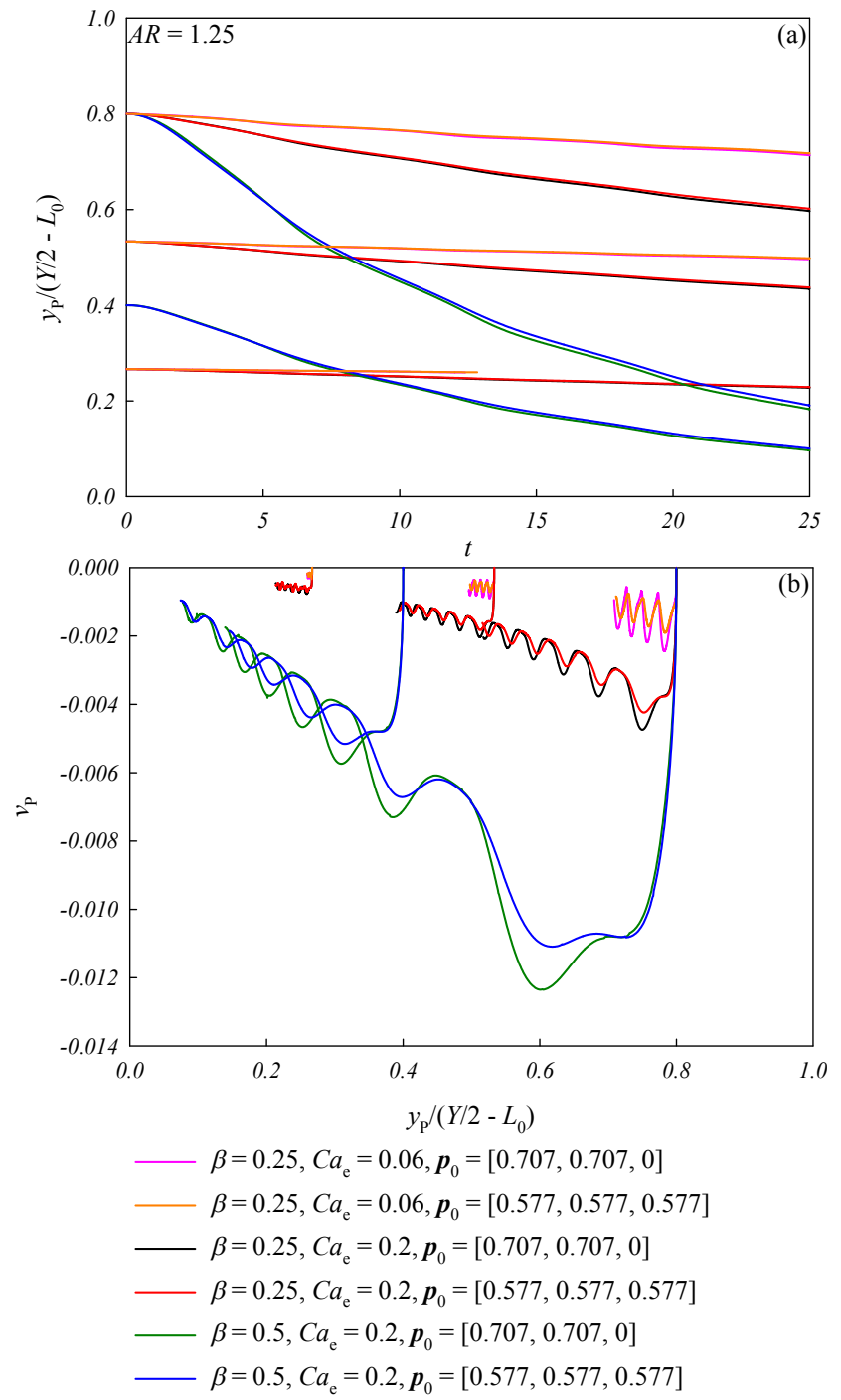

FIG. 10. (Color online) a) Trajectories of a neo-Hookean elastic prolate spheroid with aspect ratio $A R=1.25$ in a Newtonian fluid under confined shear flow; b) Migration velocity $v_{\mathrm{P}}$ of a neo-Hookean elastic prolate spheroid in a Newtonian fluid under confined shear flow as a function of its normalized vertical position $y_{\mathrm{P}} /\left(Y / 2-L_{0}\right)$. Both panels show results at varying blockage ratio $\beta$, elastic capillary number $C a_{\mathrm{e}}$, initial orientation $\boldsymbol{p}_{0}$, and initial vertical position of the particle $y_{\mathrm{P} 0} /\left(Y / 2-L_{0}\right)$.

shape (see Fig. 12a).

When the spheroid is suspended asymmetrically with respect to the walls, and, in particular, it is quite close to one of them, its deformed shape is no longer ellipsoidal. In order to visualize this better, in Fig. 11, 5 front $(x y-)$ and top $(x z-)$ views are reported from the dynamics of a particle with aspect ratio $A R=1.25$, initial orientation $p_{0}=[0.577,0.577,0.577]$, initial position $y_{\mathbf{P O}} /\left(Y / 2-L_{0}\right)=0.8$, blockage ratio $\beta=0.5$, 


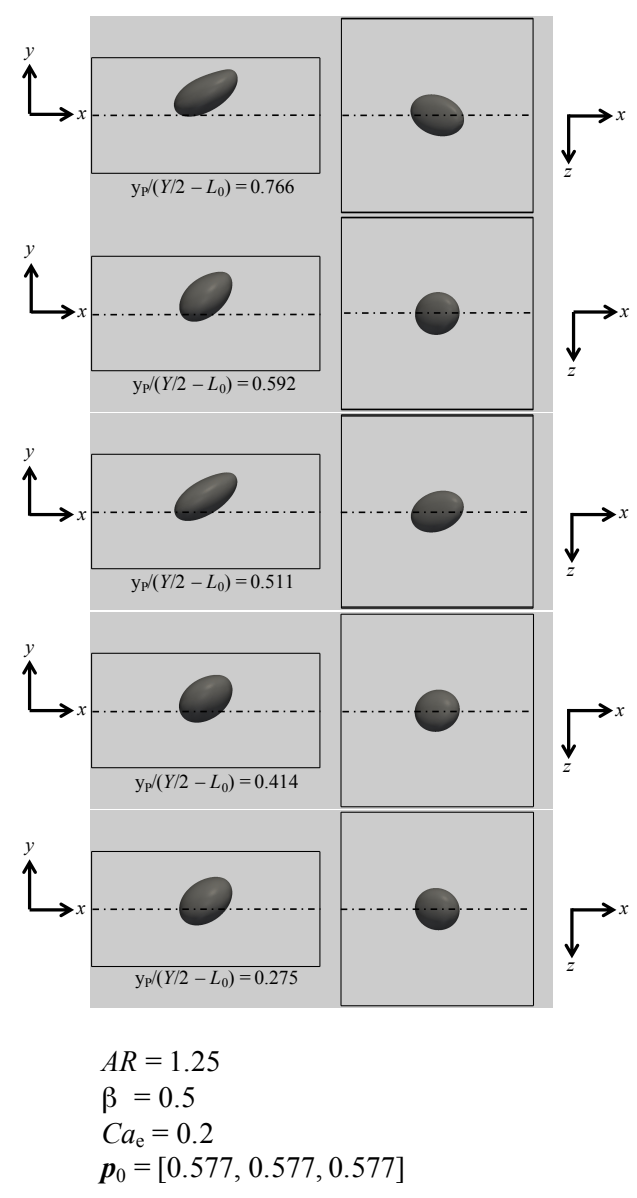

FIG. 11. Dynamics of a neo-Hookean elastic prolate spheroid with aspect ratio $A R=1.25$, initial orientation $\boldsymbol{p}_{0}=[0.577,0.577,0.577]$, initial position $y_{\mathrm{P} 0} /\left(Y / 2-L_{0}\right)=$ 0.8 , blockage ratio $\beta=0.5$, and elastic capillary number $C a_{\mathrm{e}}=0.2$. The shapes attained by the particle at five vertical positions are displayed, i.e., $y_{\mathrm{P}} /\left(Y / 2-L_{0}\right)=$ $0.766,0.592,0.511,0.414,0.275$. Left: front views (the dashdot line is the trace of the $x z$-plane at $y=0$ ); right: top views (the dash-dot line is the trace of the $x y$-plane at $z=0$ ).

and elastic capillary number $C a_{\mathrm{e}}=0.2$. On the left, the first view, at $y_{\mathbf{P}} /\left(Y / 2-L_{0}\right)=0.766$, shows that the particle, released quite close to the upper wall of the flow cell, deforms asymmetrically, attaining a slipper-like shape; then, going through the snapshots on the left, it can be observed that, as the particle migrates towards the channel center plane (whose trace is given by the dash-dot line), its shape and orientation go through oscillations. Moreover, the top views on the right show that, during its dynamics, the particle shape is not symmetric neither with respect to the shear plane (whose trace is given by the dash-dot line).

Even if the deformed shapes displayed in Fig. 11 are not ellipsoidal, it is evident that such shapes are still 'smooth' figures with a preferential orientation. Hence, in order to give a quan- titative description of the deformation and orientation of a particle out of the shear plane, we consider a box encasing it. The semi-dimensions $L, B$, and $W$ of such box are analogous to the semiaxes of an ellipsoid, and the unit vector $p$ oriented as the major dimension $L$ is similar to the orientation unit vector of an ellipsoid.
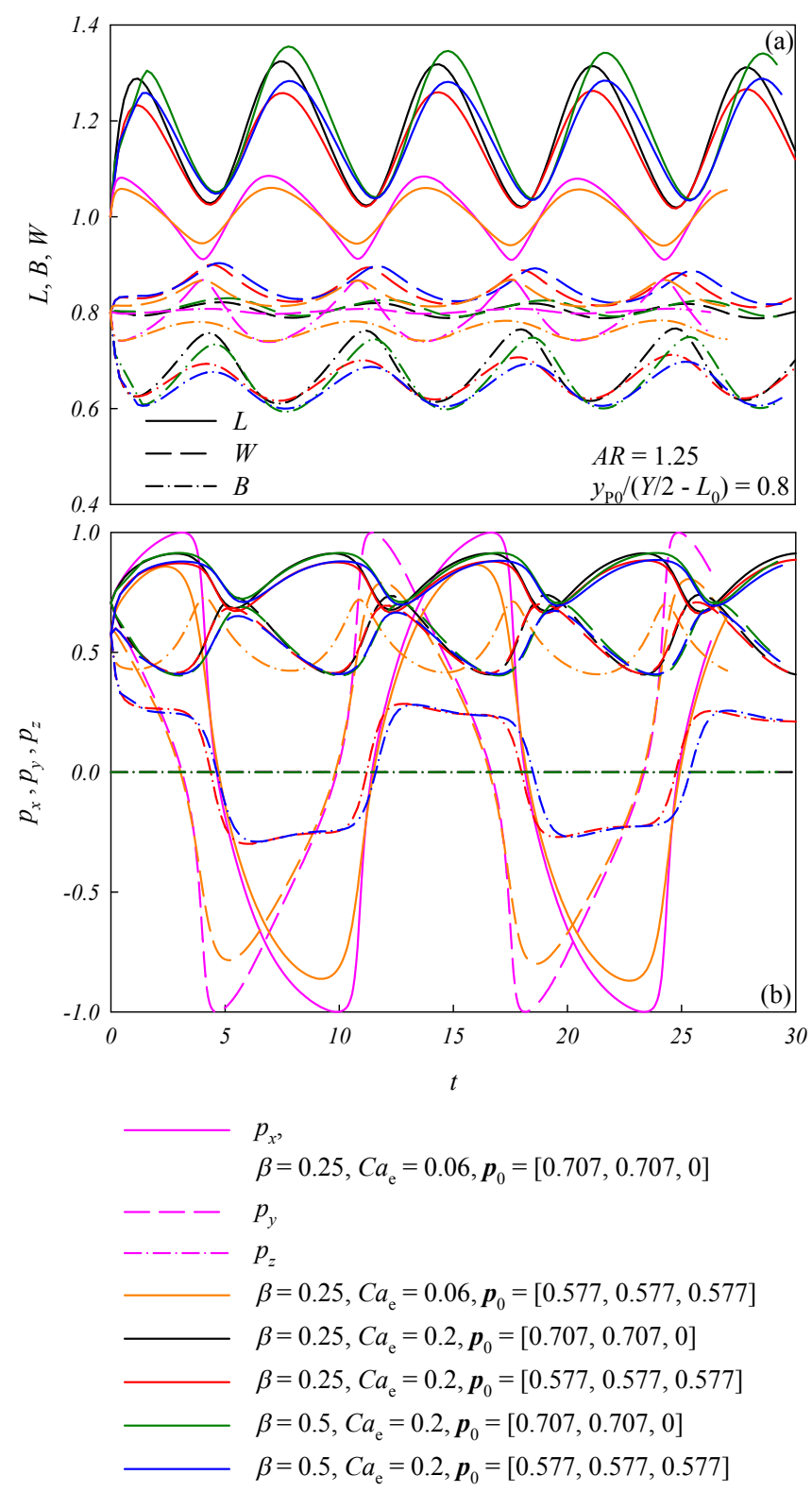

FIG. 12. (Color online) Dynamics of a neo-Hookean elastic prolate spheroid with aspect ratio $A R=1.25$ suspended with initial vertical position $y_{\mathrm{P} 0} /\left(Y / 2-L_{0}\right)=0.8$ in a Newtonian fluid under confined shear flow. a) Time evolution of the dimensions $L, B, W$; b) Time evolution of the Cartesian components of the orientation unit vector $\boldsymbol{p}$. Both panels show results at varying blockage ratio $\beta$, elastic capillary number $C a_{\mathrm{e}}$, and initial orientation $\boldsymbol{p}_{0}$. 
Fig. 12a displays the temporal trends of $L$ (solid curves), $W$ (dashed curves), and $B$ (dash-dot curves) for a particle with aspect ratio $A R=1.25$ suspended with initial vertical position $y_{\mathbf{P 0}} /(Y / 2-$ $\left.L_{0}\right)=0.8$ for the same parameters as in Fig. 10 . For all the sets of parameters considered, it can be seen that, after an initial transient, the lengths of the three semiaxes oscillate with defined frequency. However, at variance with the cases shown in Figs. 2a, 5a, and 7a, where the particle is placed in the middle of the channel gap, the amplitude of such oscillations is not constant. This is due to the interplay of deformation and migration, because the particle 'feels' a changing stress while it goes away from the wall; as it reaches the channel center plane, the oscillations of $L, W$, and $B$ will attain the same (constant) frequency and amplitude of the ones for a particle starting at $y_{P 0}=0$ with the same $\beta$ and $C a_{\mathbf{e}}$.

Fig. $12 \mathrm{~b}$ reports the temporal trends of the Cartesian components $p_{x}$ (solid lines), $p_{y}$ (dashed lines), and $p_{z}$ (dash-dot lines) of the orientation vector of the particle $p$. When the spheroid is initially oriented within the shear plane, i.e., with $\boldsymbol{p}_{0}=[0.707,0.707,0]$ (see the pink, black, and green curves), the orientation vector always lies on such plane, so $p_{z}$ is always nil. On the contrary, $p_{x}$ and $p_{y}$ oscillate, and a qualitative difference can be detected between the case at $C a_{\mathbf{e}}=0.06$ (pink curves) and the case at $C a_{\mathbf{e}}=0.2$ (black and green curves). Indeed, for $C a_{\mathbf{e}}=0.06, p_{x}$ and $p_{y}$ oscillate between -1 and +1 , then the particle major semiaxis performs periodical revolutions on the shear plane, whereas, for $C a_{\mathrm{e}}=0.2$, the $x$ - and $y$-components of $\boldsymbol{p}$ oscillate always assuming positive values; in other words, the particle major semiaxis forms an angle with the flow direction that is always positive. Two similar behaviors had been already detected for particles lying on the shear plane and starting on the channel center plane (see Figs. 2b, 5b, and 7b): in particular, the first is a TU-like behavior, whereas the second is a TR-like behavior. For $\beta=0.25$ and $C a_{\mathbf{e}}=0.06$, when the particle is initially oriented out of the shear plane, i.e., with $\boldsymbol{p}_{0}=[0.577,0.577,0.577]$ (orange curves), the $x$ - and $y$-components of its orientation vector $p$ oscillate with increasing peak heights, whereas its $z$-component oscillates with decreasing average and peak heights, always staying positive. As time goes by, then, the particle orientational behavior will tend to conform to the one of the particle starting on the shear plane with the same parameters (pink curve). For $\beta=0.25,0.5$ and $C a_{\mathbf{e}}=0.2$, when the particle is initially oriented out of the shear plane with $\boldsymbol{p}_{0}=[0.577,0.577,0.577]$ (red and blue curves), from the $p_{x^{-}}$and $p_{y}$-trends it is evident that the particle major semiaxis oscillates always staying above the flow-vorticity plane, whereas, from the $p_{z}$-trend, it can be seen that, during its oscillations, the particle continuously crosses the flowgradient plane. It is visible in Fig. $12 \mathrm{~b}$ that the $p_{z}$-oscillations around $p_{z}=0$ tend to damp, thus, at long times, the orientational behaviors of the particles starting out of the shear plane will conform to the ones of the particles starting on the plane for the same values of the other parameters.

In order to give a better insight into particle orientation dynamics, in Fig. 13 the projections on the flow-gradient (a) and flow-vorticity (b) planes of the trajectories traced by the unit vector $p$ are shown for a particle with aspect ratio $A R=1.25$ suspended with initial vertical position $y_{\mathbf{P 0}} /\left(Y / 2-L_{0}\right)=0.8$ for the same parameters as in Fig. 10. The two particle initial orientations considered (i.e., $\boldsymbol{p}_{0}=[0.707,0.707,0],[0.577,0.577,0.577]$ ) are represented by empty circles, and arrows identify the directions of travel of the trajectories. The particle with $\beta=0.25$ and $C a_{\mathbf{e}}=0.06$ initially lying on the shear plane (pink curves) undergoes TU motion: by looking at panel a, it can be noticed that the projection of $p$ moves clockwise along a circular trajectory; consequently, from panel $b$ it appears that the projection of $p$ on the shear-vorticity plane goes back-and-forth along the $x$-axis. When an initial orientation out of the shear plane are considered (orange curves), the projection of $p$ on the $x y$-plane has a spirallike trajectory with increasing radius, whereas its $x z$-projection oscillates drawing 'horizontal 8s' with increasing amplitude and decreasing $z$ component. At long times, the dynamics of a particle starting out of the shear plane will conform to TU. For $C a_{\mathbf{e}}=0.2, \beta=0.25,0.5$, the particle with $\boldsymbol{p}_{0}=[0.707,0.707,0]$ (black and green curves) follows the aforementioned TR motion: by looking at panel a, it can be seen that the projection of $\boldsymbol{p}$ on the $x y$-plane goes back-and-forth along the same trajectory; similarly, by looking at panel b, it can be observed that the projection of $p$ on the $x z$-plane goes back-and-forth on a portion of the $x$-axis. When the initial orientation out of the shear plane $\boldsymbol{p}_{0}=[0.577,0.577,0.577]$ is considered (red and blue curves), after an initial transient, the $x y$-projection of $\boldsymbol{p}$ 'draws' arcs of increasing amplitude, whereas its $x z$-projection traces 'vertical 8-s' of decreasing amplitude symmetric about the $x$-axis. At long times, the dynamics of particles starting out of the shear plane will conform to the TR motion of spheroids initially placed with the major axis on the shear plane.

We are, then, in the position to say that a neoHookean elastic prolate spheroid suspended in a Newtonian fluid under shear flow with its center of volume initially placed in an asymmetric position with respect to the two moving walls of the 

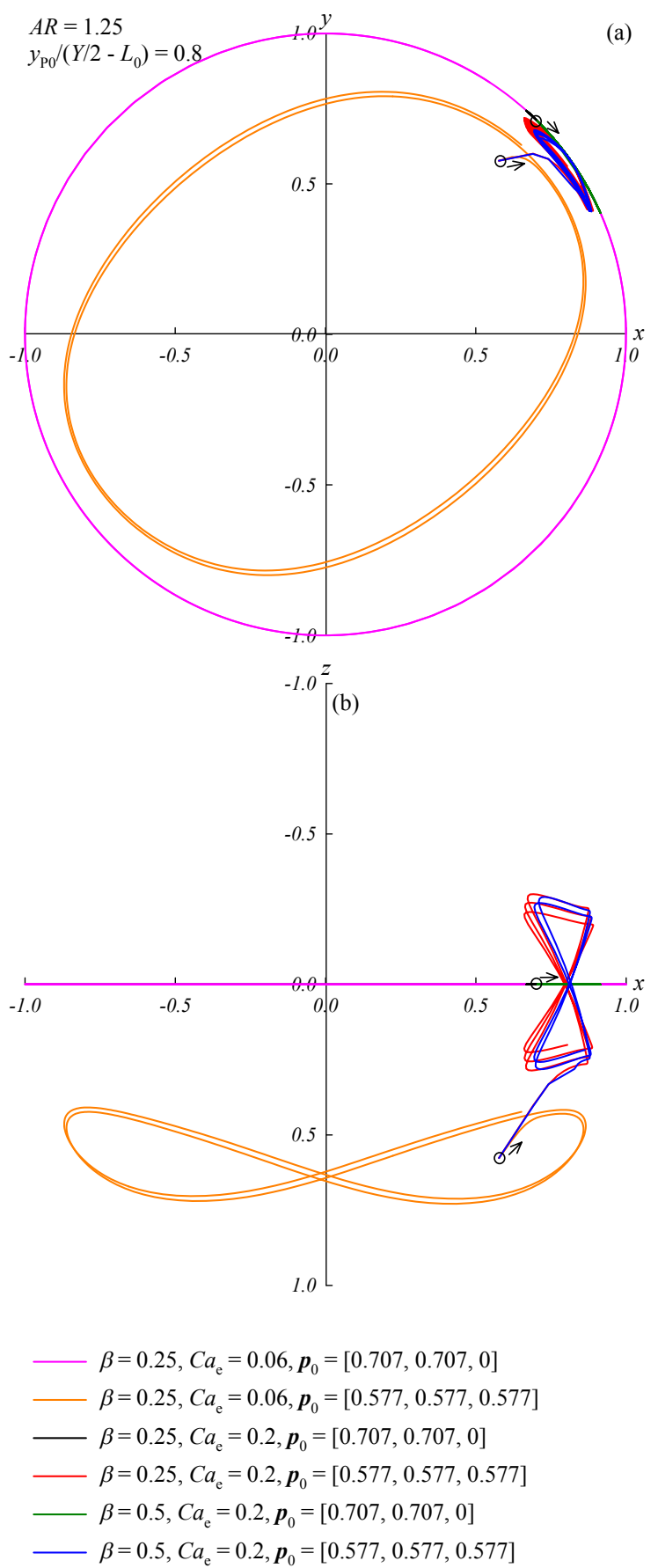

FIG. 13. (Color online) Dynamics of a neo-Hookean elastic prolate spheroid with aspect ratio $A R=1.25$ suspended with initial vertical position $y_{\mathrm{P} 0} /\left(Y / 2-L_{0}\right)=0.8$ in a Newtonian fluid under confined shear. a) Projection on the $x y$-plane of the trajectories traced by the orientation unit vector $\boldsymbol{p}$; b) Projection on the $x z$-plane of the trajectories traced by the orientation unit vector $\boldsymbol{p}$. Both panels show results at varying blockage ratio $\beta$, elastic capillary number $C a_{\mathrm{e}}$, and initial orientation $\boldsymbol{p}_{0}$.

flow cell experiences two concomitant phenom- ena, namely, unsteady deformation and lateral migration towards the center plane of the flow cell. For what matters particle shape deformation and orientation, like in the case of a prolate spheroid suspended on the center plane of the flow cell, fixed the aspect ratio $A R$, 'low' values of the elastic capillary number $C a_{\mathbf{e}}$ promote $\mathrm{TU}$, whereas 'high' $C a_{\mathrm{e}}$-values promote TR. Particles initially oriented out of the shear plane will come to the shape and orientation behavior of particles initially oriented within the shear plane after passing through more complicated dynamics. Lateral migration superimposes to these phenomena: in a Newtonian fluid, a neo-Hookean elastic prolate spheroid always migrates towards the channel center plane, with a (oscillating) velocity increasing (in magnitude) both with $\beta$ and $C a_{\mathbf{e}}$.

\section{CONCLUSIONS}

In this paper, we investigate through numerical simulations the dynamics of a neo-Hookean elastic prolate spheroid suspended in a Newtonian fluid under shear flow. Both initial orientations of the particle within and outside the shear plane and both unbounded and confined flow geometries are considered. For confined flow, we consider also asymmetric initial positions of the particle with respect to the moving walls.

The dimensionless parameters that govern the system are the particle aspect ratio $A R$, which is the ratio of the major and the minor spheroid semiaxes, the elastic capillary number $C a_{\mathrm{e}}$, that relates the viscous forces acting on the particle and its elasticity, and the blockage ratio $\beta$, that relates the particle and the flow cell characteristic dimensions.

In unbounded flow, when the particle starts on the shear plane, two stable regimes of motion are found, namely, trembling (TR), where the particle periodically elongates and compresses and the angle between its major semiaxis and the flow direction oscillates around a mean positive value, and tumbling (TU), where the particle shape periodically changes and its major axis performs complete revolutions around the vorticity axis. Higher $A R$-values promote TU, whereas higher $C a_{\mathrm{e}}$-s promote TR.

When the particle is initially oriented out of the shear plane, more complex dynamics are detected. However, such behaviors are 'transient', since they evolve in time towards TR or TU, i.e., $p_{z} \rightarrow 0$ for $t \rightarrow \infty$, where $p_{z}$ is the component in the vorticity direction of the unit vector that gives particle orientation.

The presence of solid walls in the vicinity of the particle influences its deformation and regime of motion. More confined geometries, i.e., higher $\beta$-s, promote TR.

A phase diagram that summarizes the effects of the three parameters that rule the system is presented, and our numerical results are compared with the non- 
perturbative calculations from Gao et. al [16] for elastic prolate spheroids in a Newtonian fluid under unbounded shear flow, yielding a complete quantitative agreement.

When the spheroid center of volume is initially placed in an asymmetric position with respect to the two moving walls of the flow cell, the particle undergoes two concomitant phenomena, namely, unsteady deformation and lateral migration to- wards the center plane of the flow cell. Concerning particle deformation and orientation, the same qualitative behaviors described above for particles symmetrically suspended between the walls are detected. Lateral migration superimposes to these phenomena, with a (oscillating) migration velocity increasing (in magnitude) both with $\beta$ and $C a_{\mathbf{e}}$.
[1] R. Roscoe, J. Fluid Mech. 28, 273 (1967).

[2] T. Gao and H. H. Hu, J. Comput. Phys. 228, 2132 (2009).

[3] K. Sugiyama, S. Ii, S. Takeuchi, S. Takagi, and Y. Matsumoto, J. Comput. Phys. 230, 596 (2011).

[4] T. Gao, H. H. Hu, and P. P. Castañeda, J. Fluid Mech. 687, 209 (2011).

[5] J. D. Eshelby, Proc. R. Soc. Lond. A 241, 376 (1957).

[6] J. D. Eshelby, Proc. R. Soc. Lond. A 252, 561 (1959).

[7] M. M. Villone, M. A. Hulsen, P. D. Anderson, and P. L. Maffettone, Comput. Fluids 90, 88 (2014).

[8] M. M. Villone, F. Greco, M. A. Hulsen, and P. L. Maffettone, J. Non-Newtonian Fluid Mech. 210, 47 (2014).

[9] G. B. Jeffery, Proc. R. Soc. Lond. A 102, 161 (1922).

[10] H. L. Goldsmith and S. G. Mason, The microrheology of dispersions, Rheology: Theory and Applications, Vol. 4 (Academic Press Inc., 1967).

[11] P. L. Frattini and G. G. Fuller, J. Fluid Mech. 168, 119 (1986).

[12] S. J. Johnson and G. G. Fuller, J. Colloid Interface Sci. 124, 441 (1988).
[13] J. Vermant, H. Yang, and G. G. Fuller, AIChE J. 47, 790 (2001).

[14] D. Abreu, M. Levant, V. Steinberg, and U. Seifert, Adv. Colloid Interface Sci. 208, 129 (2014).

[15] R. Finken, S. Kessler, and U. Seifert, J. Phys.: Condens. Matter 23, 184113 (2011).

[16] T. Gao, H. H. Hu, and P. P. Castañeda, Phys. Rev. Lett. 108, 058302 (2012).

[17] R. Guénette and M. Fortin, J. Non-Newtonian Fluid Mech. 60, 27 (1995).

[18] A. C. Bogaerds, A. M. Grillet, G. W. Peters, and F. P. Baaijens, J. Non-Newtonian Fluid Mech. 108, 187 (2002).

[19] A. N. Brooks and T. J. Hughes, Comp. Meth. Appl. Mech. Eng. 32, 199 (1982).

[20] D. Cordasco and P. Bagchi, Phys. Fluids 25, 091902 (2013).

[21] G. D'Avino, M. Hulsen, F. Greco, and P. L. Maffettone, Phys. Rev. E 89, 043006 (2014). 